\title{
SEGURANÇA ALIMENTAR DOS ESTABELECIMENTOS COMERCIAIS DE ALIMENTAÇÃO DO MUNICÍPIO DE CAMPINAS E REGIÃO: SÃO PAULO, VERIFICAÇÃ̃O DOCUMENTAL
}

\author{
Alimentary security of the commercial food establishments located at Campinas and \\ region: documentary verification \\ Eduarda Alonso Mazziero ${ }^{1}$, Vanessa Fernandes Coutinho², Renato Ribeiro Nogueira \\ Ferraz $^{3}$ \\ ${ }^{1-3}$ Universidade Estácio de Sá - SP
}

\section{RESUMO}

A segurança alimentar e nutricional está diretamente relacionada ao aproveitamento total dos estabelecimentos de alimentação das boas práticas de higiene e manipulação. No Brasil, a regulamentação das Boas Práticas de Alimentação é regulamentada pela Agência Nacional de Vigilância Sanitária (ANVISA). Entre os principais documentos utilizados, estão a CVS 5/2013, a RDC 216/2004. A qualidade nutricional do alimento, está diretamente relacionada ao seguimento dessas normas. O presente trabalho visa conhecer a realidade de estabelecimentos comerciais de alimentação do município de Campinas e região - São Paulo, verificando se os mesmos possuem as documentações exigidas pelos órgãos fiscalizadores. Trata-se de um estudo observacional de abordagem qualitativa. A coleta de dados foi realizada em estabelecimentos comerciais na cidade de Campinas e região - São Paulo no segundo semestre de 2017. Foram entrevistados um total de 31 estabelecimentos e as perguntas realizadas foram: quantos anos o estabelecimento possui de funcionamento, se possui alvará de funcionamento, se possui licença de funcionamento, se já recebeu visita da vigilância sanitária e se sim qual foi o motivo (inicial para liberação de licença, rotina ou denúncia). Diante dos dados apresentados foi possível verificar que a maioria dos estabelecimentos entrevistados tem mais que 10 anos de funcionamento e possuem o alvará e licença de funcionamento, sendo também a maioria deles já visitados pela vigilância sanitária em algum momento. Foi possível concluir que a maioria dos estabelecimentos entrevistados possui as documentações principais que asseguram que o estabelecimento está apto a funcionar e vender/manipular alimentos e que a segurança alimentar fica diretamente relacionada a legalidade desses estabelecimentos.

Palavras-chave: Segurança alimentar, estabelecimentos comerciais de alimentação, vigilância sanitária, legislação, boas práticas.

\begin{abstract}
Food and nutritional security is directly related to the total use of food establishments for good hygiene and handling practices. In Brazil, the regulation of Good Food Practices is regulated by the National Health Surveillance Agency (ANVISA). Among the main documents used are CVS 5/2013, RDC 216/2004. The nutritional quality of the food is directly related to the follow-up of these standards. The present work aims to know the reality of commercial food establishments in the city of Campinas and region - São Paulo, verifying if they have the documentation required by the inspection agencies. This is an observational study with a qualitative approach. Data collection was carried out in commercial establishments in the city of Campinas and region - São Paulo in the second half of 2017. A total of 31 establishments were interviewed and the questions asked were: how many years the establishment has operating, if it has operating license, if you have
\end{abstract}


an operating license, if you have already visited the sanitary surveillance and if for what reason (initial release of license, routine or report). Based on the data presented, it was possible to verify that most of the establishments interviewed have more than 10 years of operation and have the license and operating license, most of them already visited by sanitary surveillance at some point. It was possible to conclude that most of the interviewed establishments have the main documents that ensure that the establishment is able to function and sell / handle food and that food security is directly related to the legality of these establishments.

Keywords: Food security, commercial food establishments, sanitary surveillance, legislation, good practices

\section{INTRODUÇÃO}

A segurança alimentar e nutricional está diretamente relacionada ao aproveitamento total dos estabelecimentos de alimentação das boas práticas de higiene e manipulação. Por conta das diversas mudanças adotadas pela sociedade moderna, entre elas as jornadas de trabalho mais duradouras e/ou ainda a regularidade notória de comer fora de casa, tornou-se rotineiro a ida até um desses estabelecimentos, trazendo assim as preocupações abordadas (CAVALLI, SALAY, 2007; SALAY, 2005; CUNHA, MAGALHÃES, BONAS, 2013).

As práticas alimentares dos indivíduos que se alimentam fora do domicílio se moldam conforme a relação construída com o lugar e tipo de alimento que é consumido. Os que precisam fazer isso com frequência mostram preocupação com a qualidade do serviço de alimentação, levando em conta como a refeição é preparada, ou tipo de ingredientes que são utilizados, se há alguma forma de fiscalização e controle, entre outras questões (GARCIA, 1997).

No Brasil, a regulamentação das Boas Práticas de Alimentação é regulamentada pela Agência Nacional de Vigilância Sanitária (ANVISA), através de publicações oficiais para nortear os trabalhos dos profissionais da área. Entre os principais documentos utilizados, estão a CVS 5/2013, a RDC 216/2004, além de outros documentos que tratam de situações específicas de boas práticas de fabricação de alguns alimentos, rotulagem e informação nutricional, entre outros. A ANVISA possui protocolos específicos para emitir as permissões e documentos necessários para que os estabelecimentos comerciais funcionem, porém, a teoria muitas vezes não consegue acompanhar a realidade da pratica. A qualidade nutricional do alimento, sendo uma das principais observações a ser analisada, está diretamente relacionada ao seguimento dessas normas (BRASIL, 2013; BRASIL, 2004; CAVALLI, SALAY, 2007).

A qualidade higiênico-sanitária dos alimentos e dos estabelecimentos de alimentação vem sendo amplamente discutida e estudada, por conta de sua importância no desenvolvimento e veiculação de Doenças Transmitidas por Alimentos (DTA), sendo essas um dos principais contribuintes e protagonistas dos aumentos de índices de mortalidade nos países subdesenvolvidos, sendo considerado pelos principais órgãos de saúde como um dos maiores problemas de saúde pública da atualidade (OMS, 2017; CUNHA, MAGALHÃES, BONNAS, 2013).

Sendo assim, levando em conta os dados apresentados e a importância do monitoramento da qualidade nutricional e segurança alimentar dos estabelecimentos comerciais de alimentação, o presente trabalho visa conhecer a realidade de estabelecimentos comerciais de alimentação do município de Campinas e região - São Paulo, verificando se os mesmos possuem as documentações exigidas pelos órgãos fiscalizadores e se sofrem ou não essa fiscalização na prática. 


\section{OBJETIVO}

Conhecer a realidade dos estabelecimentos comerciais de alimentação do município de Campinas e região - SP, por meio da verificação de documentações necessárias exigidas pelos órgãos fiscalizadores para o funcionamento, como o preconizado pela legislação.

\section{MÉTODO}

Trata-se de um estudo observacional de abordagem qualitativa. A coleta de dados foi realizada em estabelecimentos comerciais na cidade de Campinas e região - SP no segundo semestre de 2017. Os estabelecimentos entrevistados são clientes de uma empresa de consultoria e assessoria terceirizada formada por uma equipe de nutricionistas e trabalham com a finalidade de melhorias da qualidade higiênico-sanitária. Foram entrevistados os estabelecimentos onde no dia da visita realizada pela empresa de consultoria e assessoria estavam presente o dono ou responsável, sendo as perguntas de fácil entendimento, onde qualquer pessoa com o conhecimento da documentação do estabelecimento poderia responder.

As perguntas realizadas foram: quantos anos o estabelecimento possui de funcionamento, se possui alvará de funcionamento, se possui licença de funcionamento, se já recebeu visita da vigilância sanitária e se sim qual foi o motivo (inicial para liberação de licença, rotina ou denúncia). Essas perguntas tiveram a função de explorar as características do estabelecimento e verificar se o mesmo possui toda a documentação preconizada pelos órgãos fiscalizadores e se já sofreram algum tipo de visita de fiscalização. Este trabalho atendeu a resolução 466/2012 com relação aos aspectos éticos de pesquisas envolvendo seres humanos.

\section{RESULTADOS}

Foram entrevistados os responsáveis de 31 estabelecimentos, dentre eles estão restaurantes e bares, padarias, mercados, fábrica de pão de queijo e revenda de espetos. Os estabelecimentos são da cidade de Campinas, Paulínia, Valinhos, Sumaré, Louveira e Indaiatuba-SP. Os resultados estão descritos na tabela abaixo. 
Tabela 1: Informações documentais dos estabelecimentos comerciais de alimentação do município de Campinas e região, 2017.

\begin{tabular}{|c|c|c|c|c|c|c|c|c|c|c|c|c|}
\hline \multirow[t]{2}{*}{ Estabelecimentos } & \multicolumn{3}{|c|}{ Quantos anos o estabelecimento possui? } & \multicolumn{2}{|c|}{ Possui alvará de uso? } & \multicolumn{2}{|c|}{$\begin{array}{l}\text { Possui licença de } \\
\text { funcionamento? }\end{array}$} & \multicolumn{2}{|c|}{$\begin{array}{l}\text { Já recebeu visita da } \\
\text { vigilância sanitária? }\end{array}$} & \multicolumn{3}{|c|}{ Tipo de visita } \\
\hline & $\begin{array}{c}\text { Menos que } 5 \\
\text { anos }\end{array}$ & 5 e 10 anos & $\begin{array}{l}\text { Mais que } 10 \\
\text { anos }\end{array}$ & Sim & Não & Sim & Não & Sim & Não & $\begin{array}{c}\text { Inicial } \\
\text { Liberação de } \\
\text { licença }\end{array}$ & Rotina & Denúncia \\
\hline 1 & & $\mathrm{X}$ & & $\mathrm{X}$ & & $\mathrm{X}$ & & $\mathrm{X}$ & & $\mathrm{X}$ & & \\
\hline 2 & $\mathrm{X}$ & & & $\mathrm{X}$ & & $X$ & & & $\mathrm{X}$ & & & \\
\hline 3 & & & $\mathrm{X}$ & $\mathrm{X}$ & & $\mathrm{X}$ & & $\mathrm{X}$ & & & & $\mathrm{X}$ \\
\hline 4 & $\mathrm{X}$ & & & $\mathrm{X}$ & & & $X$ & $\mathrm{X}$ & & $\mathrm{X}$ & $\mathrm{X}$ & \\
\hline 5 & & & $\mathrm{X}$ & $\mathrm{X}$ & & $X$ & & $\mathrm{X}$ & & $\mathrm{X}$ & $X$ & \\
\hline 6 & $\mathrm{X}$ & & & & $\mathrm{X}$ & & $\mathrm{X}$ & $X$ & & $\mathrm{X}$ & & \\
\hline 7 & & & $\mathrm{X}$ & $\mathrm{X}$ & & & $\mathrm{X}$ & & $\mathrm{X}$ & & & \\
\hline 8 & & $\mathrm{X}$ & & $\mathrm{X}$ & & $\mathrm{X}$ & & $\mathrm{X}$ & & & $\mathrm{X}$ & \\
\hline 9 & $\mathrm{X}$ & & & & $\mathrm{X}$ & & $\mathrm{X}$ & $\mathrm{X}$ & & $\mathrm{X}$ & & \\
\hline 10 & & & $\mathrm{X}$ & & $\mathrm{X}$ & & $\mathrm{X}$ & & $\mathrm{X}$ & & & \\
\hline 11 & $\mathrm{X}$ & & & & $\mathrm{X}$ & & $\mathrm{X}$ & & $\mathrm{X}$ & & & \\
\hline 12 & $\mathrm{X}$ & & & $\mathrm{X}$ & & & $X$ & & $\mathrm{X}$ & & & \\
\hline 13 & $\mathrm{X}$ & & & $\mathrm{X}$ & & $\mathrm{X}$ & & & $\mathrm{X}$ & & & \\
\hline
\end{tabular}

\begin{tabular}{|c|c|c|c|c|c|c|c|c|c|c|c|c|}
\hline 14 & & $\mathrm{X}$ & & & $\mathrm{X}$ & & $\mathrm{X}$ & & $X$ & & & \\
\hline 15 & & & $X$ & $\mathrm{X}$ & & $\mathrm{X}$ & & $X$ & & & $\mathrm{X}$ & \\
\hline 16 & $X$ & & & & $\mathrm{X}$ & & $\mathrm{X}$ & & $\mathrm{X}$ & & & \\
\hline 17 & $X$ & & & & $\mathrm{X}$ & & $\mathrm{X}$ & & $\mathrm{X}$ & & & \\
\hline 18 & & & $\mathrm{X}$ & $\mathrm{X}$ & & $\mathrm{X}$ & & $\mathrm{X}$ & & $\mathrm{X}$ & & \\
\hline 19 & $X$ & & & & $X$ & & $\mathrm{X}$ & $\mathrm{X}$ & & & & $\mathrm{X}$ \\
\hline 20 & & & $\mathrm{X}$ & $\mathrm{X}$ & & $\mathrm{X}$ & & $X$ & & & $\mathrm{X}$ & \\
\hline 21 & & & $\mathrm{X}$ & $\mathrm{X}$ & & $X$ & & $\mathrm{X}$ & & & $\mathrm{X}$ & \\
\hline 22 & $X$ & & & $X$ & & & $X$ & & $\mathrm{X}$ & & & \\
\hline 23 & & & $\mathrm{X}$ & $\mathrm{X}$ & & $\mathrm{X}$ & & $\mathrm{X}$ & & & $\mathrm{X}$ & $\mathrm{X}$ \\
\hline 24 & & & $\mathrm{X}$ & $\mathrm{X}$ & & $\mathrm{X}$ & & $\mathrm{X}$ & & $\mathrm{X}$ & & $\mathrm{X}$ \\
\hline 25 & & & $\mathrm{X}$ & $\mathrm{X}$ & & $\mathrm{X}$ & & $\mathrm{X}$ & & $\mathrm{X}$ & $\mathrm{X}$ & $\mathrm{X}$ \\
\hline 26 & & $\mathrm{X}$ & & $\mathrm{X}$ & & & $\mathrm{X}$ & & $X$ & & & \\
\hline 27 & & $\mathrm{X}$ & & $\mathrm{X}$ & & $X$ & & $\mathrm{X}$ & & $\mathrm{X}$ & & $\mathrm{X}$ \\
\hline 28 & & & $\mathrm{X}$ & $\mathrm{X}$ & & $X$ & & $\mathrm{X}$ & & $\mathrm{X}$ & & \\
\hline 29 & & & $\mathrm{X}$ & $\mathrm{X}$ & & $\mathrm{X}$ & & $\mathrm{X}$ & & & $\mathrm{X}$ & \\
\hline 30 & $\mathrm{X}$ & & & & $\mathrm{X}$ & & $\mathrm{X}$ & & $X$ & & & \\
\hline 31 & & $\mathrm{X}$ & & & $\mathrm{X}$ & & $\mathrm{X}$ & $\mathrm{X}$ & & $\mathrm{X}$ & & \\
\hline TOTAL & 12 & 6 & 13 & 21 & 10 & 16 & 15 & 19 & 12 & 11 & 9 & 6 \\
\hline
\end{tabular}


A primeira pergunta realizada aos estabelecimentos foi "quantos anos o estabelecimento possui". Se o estabelecimento mudou de endereço ou foi trocado a gerência foi considerado o tempo no novo local ou a nova gerência. A pergunta foi distribuída em três faixas: menos que 5 anos, entre 5 e 10 anos e mais que 10 anos de funcionamento. Essa informação foi importante para mensurar há quanto tempo o estabelecimento se encontra em irregularidade ou não, já que o correto é o estabelecimento ter a documentação (alvará e licença de funcionamento) desde a inauguração ou pelo menos em andamento. Dentre os estabelecimentos entrevistados, 12 (39\%) possuem menos que 5 anos de funcionamento, 6 (19\%) estão em funcionamento entre 5 e 10 anos e 13 (42\%) estabelecimentos funcionam há mais que 10 anos.

Já a segunda pergunta foi em relação ao alvará de funcionamento, se o estabelecimento possui o documento ou não. Estabelecimentos com alvará em processo de andamento foram enquadrados na resposta como não possui alvará de funcionamento. A concessão dos alvarás é feita pelas prefeituras onde normalmente cada prefeitura pode definir suas regras. O correto é consultar a prefeitura antes de abrir o estabelecimento para verificar se há alguma restrição para exercer tal atividade no local. Sendo assim dos 31 estabelecimentos entrevistados, 21 (68\%) possuem alvará de funcionamento e 10 (32\%) não possuem o alvará ou estão com o documento em processo de liberação.

A terceira pergunta verificou se o estabelecimento possui a licença de funcionamento. Também foi considerado como não os estabelecimentos que estão com a licença em pendência, ou seja, em processo para a retirada da mesma. A licença de funcionamento também deve ser requerida junto a prefeitura de cada município. Dentre os municípios entrevistados, Campinas é a única cidade aderida ao SIL (Sistema Integrado Licenciamento) onde as solicitações referentes à Licença de Funcionamento Inicial, Renovação de Licença de Funcionamento e Alteração de Dados Cadastrais de Licença de Funcionamento devem ser feitas exclusivamente pelo portal eletrônico Via Rápida Empresa (VRE), isso para pessoas jurídicas. Campinas aderiu ao SIL em dezembro de 2015 (BORTOLLI, 2018). Já referente a este dado constatou-se que 16 (52\%) estabelecimentos possuem a licença de funcionamento em vigor e 15 (48\%) estabelecimentos não possuem esse documento ou estão o documento está em andamento.

A pergunta se o estabelecimento já recebeu a visita da vigilância sanitária foi a quarta. E questionou se o estabelecimento já recebeu em algum momento a visita da vigilância sanitária ou nunca recebeu a visita, presencial. Referente a pergunta anterior verificou-se que $19(61 \%)$ estabelecimentos já receberam a visita da vigilância sanitária e $12(39 \%)$ estabelecimentos nunca foram visitados.

A quinta pergunta está relacionada com a quarta, se o estabelecimento respondeu que já recebeu a visita da vigilância sanitária ela questiona qual foi o motivo da visita. A vigilância sanitária pode ir a um estabelecimento mediante três maneiras, a primeira é o tipo inicial onde a visita é realizada quando o estabelecimento deu entrada em sua licença de funcionamento e é verificado se o local está de acordo com as legislações vigentes para manter-se em pleno funcionamento e assim liberada a licença de funcionamento. $\mathrm{O}$ segundo tipo de visita é por rotina, onde a vigilância realiza de maneira aleatória a visita a fim de verificar as condições do estabelecimento e forçar o cliente a se adequar para dar entrada na documentação de licença de funcionamento, caso não possua a mesma. E a terceira é mediante denúncia, que é realizada anonimamente por algum cliente que visualizou alguma situação de perigo no local ou fez a compra de algum alimento em situação imprópria para o consumo, por exemplo. Através dessa pergunta foi possível verificar que há estabelecimentos que nunca receberam a visita da vigilância sanitária bem como estabelecimentos que já receberam visitas pelos diversos motivos em momento diferentes. 


\section{DISCUSSÃO}

A produção e venda de alimentos seguros envolve uma série de fatores, que devem ser exaltados. Tal importância deve ser dada não somente para cumprimento da legislação atual, mas também para a ocorrência de doenças transmitidas por alimentos que geralmente se desenvolve por falhas ocorridas em diversos momentos (CUNHA, MAGALHÃES E BONNAS, 2013).

Diante dos dados apresentados foi possível verificar que a maioria dos estabelecimentos entrevistados tem mais que 10 anos de funcionamento e possuem o alvará e licença de funcionamento, sendo também a maioria deles já visitados pela vigilância sanitária em algum momento. É possível fazer uma relação entre a segurança alimentar dos estabelecimentos e a situação dos mesmos perante as documentações exigidas para o funcionamento legal.

Uma pesquisa realizada em restaurantes comerciais do tipo self-service na região central de Maringá - PR avaliou as condições de higiene no preparo de alimentos através de aplicação de check list baseado na legislação e verificou que nenhum estabelecimento possuía proteção completa contra entrada de pragas e Manual de Boas Práticas (MBP) (GENTA, MAURÍCIO, MATIOLI, 2005). Esses dois itens são exemplos de condições básicas de estrutura física e documentos exigidos na retirada da licença de funcionamento e que quando não estão em conformidade resultam em riscos para a saúde do consumidor. Outra pesquisa encontrou resultados semelhantes, realizada em restaurantes comerciais da cidade do Rio de Janeiro-RJ, avaliou a adequação dos mesmos com critérios estabelecidos pela RDC 216 através de lista de verificação. O resultado foi de $100 \%$ de inconformidade nos quesitos de controle de saúde de funcionários e programas de capacitação em higiene com treinamentos periódicos registrados, itens esses que também integram a documentação exigida para retirada da licença de funcionamento (SANTOS, RANGEL, AZEVEDO, 2010).

Um estudo realizado na cidade de São José do Rio Preto - SP, obteve resultados parecidos com este trabalho. Foram avaliados 10 estabelecimentos produtores de alimentos, onde $30 \%$ não possuíam licença do órgão fiscalizador municipal compatível com a atividade realizada e $10 \%$, ou seja, um restaurante tinha a licença fora do prazo de validade (SEIXAS et al, 2008).

Irregularidades perante as documentações necessárias e consequentemente das boas práticas dos estabelecimentos podem ocorrer por diversos motivos, dentre eles a falta de informação e muitas vezes falta de recursos para investimentos ou a falta de importância dada a estes itens. Fazendo uma comparação entre os estabelecimentos entrevistados, o estabelecimento 2 tem menos que 5 anos de funcionamento e já possui toda documentação exigida, situação bem diferente do estabelecimento 7 que tem mais de 10 anos de funcionamento e só possui o alvará de funcionamento, em nenhum momento deu entrada na licença de funcionamento e nunca recebeu uma visita da vigilância sanitária. Vale ressaltar que devido ao crescimento do comércio alimentício a vigilância sanitária na maioria das vezes se encontra sobrecarregada e não consegue fazer nos estabelecimentos um trabalho preventivo, ficando a conta de somente cobrir as denúncias.

Barros et al (2011) avaliaram o perfil e o conhecimento em legislação sanitária de gerentes de restaurantes comerciais e verificou que a maioria dos restaurantes é gerenciado por funcionários contratados e que mais da metade dos gerentes não possuem o conhecimento dos itens que são citados na RDC 216 referente a Boas Práticas de Fabricação. E em relação a responsabilidade técnica nenhum possui nutricionista como RT, a maioria tem o chef de cozinha como responsável e que, na maioria deles também não eram capacitados com os temas referidos na RDC 216. 
Para efetivação da segurança alimentar é muito importante a capacitação dirigida aos colaboradores para operacionalizar sistemas de controle de qualidade. Essas capacitações, contudo, requerem custos e necessitam de profissionais da área de alimentos e nutrição para sua concretização. O motivo da não efetivação muitas vezes é a falta de recursos ou até não considerarem importante essa capacitação para o setor (CAVALLI, SALAY, 2007). Um fator positivo deste estudo é que os estabelecimentos entrevistados já são clientes de uma empresa de consultoria e assessoria que possui como objetivo melhorias higiênico-sanitárias, porém ainda é grande o número de estabelecimentos que se encontram em irregularidade perante os órgãos fiscalizadores.

\section{CONCLUSÃO}

Conclui-se que a maioria dos estabelecimentos entrevistados possui as documentações principais que asseguram que o estabelecimento está apto a funcionar e vender/manipular alimentos e que a segurança alimentar fica diretamente relacionada a legalidade desses estabelecimentos. A deficiência de fiscalização dos órgãos responsáveis favorece o quadro de irregularidades e insegurança alimentar.

\section{REFERÊNCIAS}

BARROS, A.P.N.; COSTA, T.F.; PEREIRA, S.C.L.; BRITO, M.J.M.; MONTEIRO, M.A.M.; ABREU, M.N.S. Perfil sociodemográfico, de conhecimentos administrativos, em legislação sanitária de gerentes e segurança alimentar em unidades produtoras de refeições comerciais. Rev. Soc. Food Nutr., v. 36, n. 2, p. 61-76, 2011.

BORTOLLI, A.A.T.Z. Vigilância em Saúde. Coordenadoria Vigilância Sanitária. Informe sobre o Licenciamento VISA, Campinas. Disponível em: http://www.saude.campinas.sp.gov.br/vigilancia/vig_sanitaria.htm.

BRASIL. Agência Nacional de Vigilância Sanitária. Resolução RDC 216, de 15 de setembro de 2004. Disponível em: http://portal.anvisa.gov.br/documents/33916/388704/RESOLU\%25C3\%2587\%25C3\%2 5830-

RDC\%2BN\%2B216\%2BDE\%2B15\%2BDE\%2BSETEMBRO\%2BDE\%2B2004.pdf/2 3701496-925d-4d4d-99aa-9d479b316c4b.

BRASIL. Agência Nacional de Vigilância Sanitária. Portaria CVS 5, de 9 de abril de 2013. Disponível em: http://www.cvs.saude.sp.gov.br/up/PORTARIA\%20CVS5_090413.pdf.

BRASIL. Ministério da Saúde. Secretaria de vigilância em saúde. Departamento de vigilância de doenças transmissíveis. Coordenação geral de doenças transmissíveis. Surtos de doenças transmitidas por alimentos. Disponível em: http://portalarquivos.saude.gov.br/images/pdf/2017/maio/29/Apresentacao-Surtos-DTA2017.pdf

CAVALLI, S.B.; SALAY, E. Gestão de pessoas em unidades produtoras de refeições comerciais e segurança alimentar. Revista de Nutrição, v. 20, n. 06, p. 657-667, 2007.

CUNHA, F.M.F.; MAGALHÃES, M.B.H.; BONNAS, D.S. Desafios da gestão de da segurança de alimentos em unidades de alimentação de nutrição no Brasil: uma revisão. Revista contextos, v. 1, n. 24, 2013.

GARCIA, R.W.D. Práticas de comportamento alimentar no meio urbano: um estudo no centro da cidade de São Paulo. Cadernos de Saúde Pública, v. 13, n. 3, p. 455-467, 1997. GENTA, T.M.S.; MAURÍCIO, A.A.; MATIOLI, G. Avaliação das boas práticas através de check-list aplicado em restaurantes self-service da região central de Maringá, Estado do Paraná. Acta Scientarum Health Sciences, v. 27, n. 2, p. 151-156, 2005. 
SEIXAS, F.R.F.; SEIXAS, J.R.F.; REIS, J.A.; HOFFMANN, F.L. Check-list para diagnóstico inicial das Boas Práticas de Fabricação (BPF) em estabelecimentos produtores de alimentos da cidade de São José do Rio Preto (SP). Revista Analytica, n. 33, 2008.

SALAY, E. Consumo alimentar fora do domicílio: implicações para pesquisas em segurança alimentar e nutricional. Disponível em: http://www.comciencia.br/dossies-172/reportagens/2005/09/14_impr.shtml

SANTOS, M.O.B.; RANGEL, V.P.; AZEVEDO, D.P. Adequação de restaurantes comerciais às Boas Práticas. Higiene Alimentar, v. 24, n. 190/191, 2010. 